\title{
Disrupters of the thymidylate synthase homodimer accelerate its proteasomal degradation and inhibit cancer growth.
}

Authors: Luca Costantino ${ }^{1 \dagger}$, Stefania Ferrari ${ }^{1 \dagger}$, Matteo Santucci ${ }^{1 \dagger}$, Outi M. H. Salo-Ahen ${ }^{3 \&}$, Emanuele Carosati ${ }^{4, \mathrm{x}}$, Silvia Franchini ${ }^{1}$, Angela Lauriola ${ }^{2 \pm}$, Cecilia Pozzi $^{5}$, Gaetano Marverti ${ }^{2}$, Matteo Trande $^{1 \pm}$, Gaia Gozzi ${ }^{1}$, Puneet Saxena ${ }^{1 \#}$, Giuseppe Cannazza ${ }^{1}$, Lorena Losi ${ }^{1}$, Daniela Cardinale ${ }^{6}$, Alberto Venturelli1 ${ }^{1, £}$, Antonio Quotadamo ${ }^{1}$, Pasquale Linciano ${ }^{1}$, Remo Guerrini ${ }^{7}$, Salvatore Pacifico ${ }^{7}$, Rosaria Luciani ${ }^{1}$, Filippo Genovese ${ }^{1}$, Stefan Henrich $^{3}$, Silvia Alboni ${ }^{1}$, Nuno Santarem ${ }^{8}$, Anabela da Silva Cordeiro ${ }^{8,9}$, Elisa Giovannetti ${ }^{10,11}$, Godefridus J Peters ${ }^{12 \wedge}$, Paolo Pinton ${ }^{11}$, Alessandro Rimessi ${ }^{11}$ Gabriele Cruciani $^{4}$, Robert M. Stroud ${ }^{13, \dagger}$, Rebecca C. Wade ${ }^{3,14,15, \dagger}$, Stefano Mangani ${ }^{5 \dagger}$, Domenico D’Arca $^{2 *}$, Glauco Ponterini ${ }^{1^{*}} \&$ Maria Paola Costi ${ }^{1 *}$

\section{Affiliations:}

${ }^{1}$ Department of Life Sciences, University of Modena and Reggio Emilia, 41125 Modena, Italy.

${ }^{2}$ Department of Biomedical, Metabolic and Neural Sciences, University of Modena and Reggio Emilia, 41125 Modena, Italy.

${ }^{3}$ Molecular and Cellular Modeling Group, Heidelberg Institute for Theoretical Studies, 69118 Heidelberg, Germany.

${ }^{4}$ Department of Chemistry, Biology and Biotechnology, University of Perugia, 06123, Perugia, Italy.

${ }^{5}$ Department of Biotechnology, Chemistry and Pharmacy, University of Siena, 53100 Siena, Italy.

${ }^{6}$ Respiratory, Critical Care \& Anesthesia UCL Great Ormond Street Institute of Child Health London, U.K.

${ }^{7}$ Department of Chemical and Pharmaceutical Science, University of Ferrara, 41121 Ferrara, Italy.

${ }^{8}$ IBMC I3S, 4200-135 Porto, Portugal. 
${ }^{9}$ Department of Biological Sciences, Faculty of Pharmacy, University of Porto, Portugal

${ }^{10}$ Department of Medical Oncology, VU University Medical Center, Cancer Center Amsterdam, 1081HV, Amsterdam, The Netherlands.

${ }^{11}$ Cancer Pharmacology Lab, Fondazione Pisana per la Scienza, 56017 Pisa, Italy.

${ }^{12}$ Dept. of Medical Sciences and Laboratory for Technologies of Advanced Therapies (LTTA), University of Ferrara, 44121 Ferrara, Italy.

${ }^{13}$ Biochemistry and Biophysics Department, University of California San Francisco, San Francisco, CA 94158, USA.

${ }^{14}$ Interdisciplinary Center for Scientific Computing (IWR), Heidelberg University, 69120 Heidelberg, Germany.

${ }^{15}$ Center for Molecular Biology (ZMBH), DKFZ-ZMBH Alliance, Heidelberg University, 69120 Heidelberg, Germany.

${ }^{\&}$ current address: Pharmaceutical Sciences Laboratory/Structural Bioinformatics Laboratory, Faculty of Science and Engineering, Pharmacy/Biochemistry, Åbo Akademi University, Tykistökatu 6A, FIN-20520 Turku, Finland.

${ }^{\mathrm{X}}$ Current address: AbocaS.p.A, loc. Aboca, 52037, Sansepolcro (AR), Italy.

${ }^{ \pm}$current address: Department of Biotechnology, University of Verona, 37134 Verona.

\#current address: Excelra Knowledge Solutions, Plot No. 79, IDA Mallapur, Nacharam, Hyderabad500076 India.

${ }^{£}$ current address: Tydock Pharma srl, 41126 Modena, Italy.

$\wedge$ second affiliation Department of Biochemistry, Medical University of Gdansk, Gdansk, Poland

*To whom correspondence should be addressed: Maria Paola Costi, mariapaola.costi@ unimore.it,

Glauco Ponterini, glauco.ponterini@unimore.it, Domenico D’Arca domenico.darca@unimore.it.

†These authors contributed equally to this work as senior authors. 
$\$$ These authors contributed equally to this work as first authors.

One Sentence Summary: Small disrupter of the dimer of thymidylate synthase accelerates its proteasomal degradation and is more active than fluorouracil in mouse cancer models.

Abstract: Drugs that target human thymidylate synthase (hTS) are widely used in anti-cancer therapy. However, treatment with classical substrate-site-directed TS inhibitors induces its overexpression and the development of drug resistance. We thus pursued an alternative strategy that led to the discovery of TS-dimer disrupters that bind at the monomer-monomer interface and shift the dimerization equilibrium of both the recombinant and the intracellular protein toward the inactive monomers. We performed a structural, spectroscopic and kinetic investigation of the effects of these small molecules and the best one, E7, accelerates the proteasomal degradation of hTS in cancer cells. E7 showed a superior anticancer profile to fluorouracil in a mouse model of human pancreatic and ovarian cancer. Thus, over sixty years after the discovery of the first TS prodrug inhibitor, fluorouracil, E7 breaks the link between TS inhibition and enhanced expression in response, providing a strategy to fight drug-resistant cancers. 


\section{Introduction}

Small molecules able to target and shift the monomer-dimer equilibrium of homodimeric enzymes may alter the metabolic pathways associated with the functions of the proteins. By binding at specific pockets of the homodimeric protein interface, they may perturb to the limit of disrupting the dimeric assembly. Such a drastic structural change of the protein may open the way to unexpected events, such as a higher liability to degradation. The causal relationship between the two events in cancer cells is still unexplored. Human thymidylate synthase (hTS) is an obligate, stable homodimer with a dimer dissociation constant of $80 \mathrm{nM}$ (1) and two active sites, each including residues from both monomers (2). As a homodimer, it provides the sole de-novo pathway to deoxythymidylate (dTMP) synthesis in human cells by catalyzing the reductive methylation of deoxyuridylate (dUMP) to dTMP using methylenetetrahydrofolate (MTHF) as the one-carbon methyl donor (2,3). By interacting with its own and other mRNAs, this protein regulates its own levels and those of other proteins involved in apoptotic processes including bcl2, c-myc, and p53 (4,5). Its inhibition is usually achieved with compounds that bind at the protein active-site, competing either with the dUMP substrate, such as 5fluorodeoxyurdine 5'-monophosphate (FdUMP), or with the folate cofactor, such as raltitrexed (RTX) and pemetrexed (PMX) (6) (4) (Fig.1a,b). However, these drugs induce cells to develop drug resistance associated with increased hTS levels which leads to therapy failure (7). A drastic change of strategy, based on the design of new compounds with different mechanisms of action, is thus necessary $(8,9)$. Here we report the discovery of molecules that bind at the hTS dimer interface and, despite their small size, shift the monomer-dimer equilibrium of the enzyme towards the inactive monomeric form. Acting as disrupters of the hTS dimer, they not only inhibit the activity of this obligate homodimeric enzyme but also favor its intracellular degradation and, hence, reduction of its level. Using a tethering approach in which sulfhydryl-containing fragments were initially identified by reaction with cysteine residues inserted by mutation around the target site, we were able to identify fragments anchored by disulfide bond formation at the interfacial mutant cysteine residues. We then 
employed molecular modeling and medicinal chemistry to modify the fragments and develop inhibitors able to bind WT hTS guided solely by their affinities for the interface region.

We provide a quantitative description of the dissociation of the hTS dimer by these small molecules and characterize the cellular protein dissociation mechanism underlying their efficacy in colon, ovarian and pancreatic cancer cells. One of our dimer disrupters, compound E7, induced apoptosis in cancer cells and a decrease of hTS levels due to enhanced proteasomal degradation of the hTS monomers with respect to the dimers. Remarkably, in a mouse model of orthotopic pancreatic cancer, this dimer disrupter caused a higher reduction of cancer growth and had lower toxicity than 5fluorouracil (5-FU), the prodrug of 5-FdUMP. Similar to what we observed in vitro, the molecular analysis of exported treated cancer tissues demonstrated decreased hTS protein and mRNA levels. These findings establish E7 as a new lead with an hTS dimer-disruptive ability and link the dimerto-monomer equilibrium shift of this protein to its faster intracellular degradation. 
A

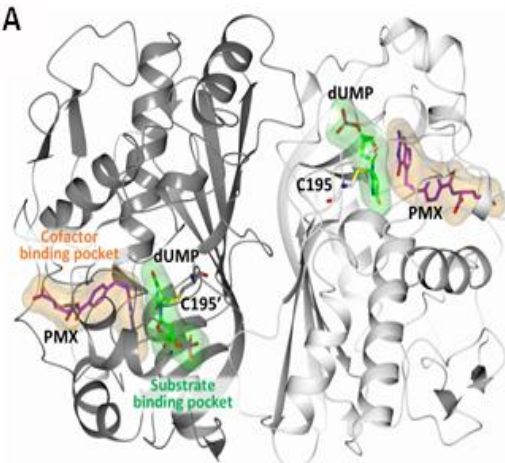

C

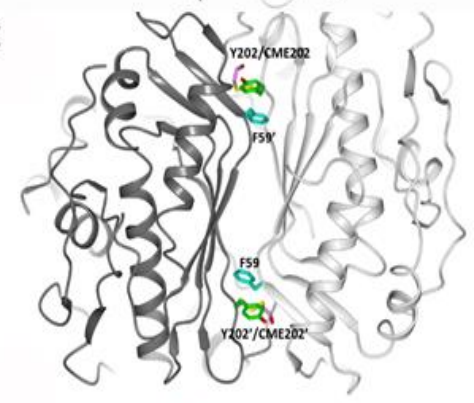

$E$

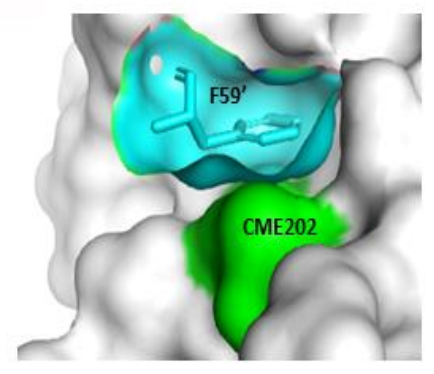

B

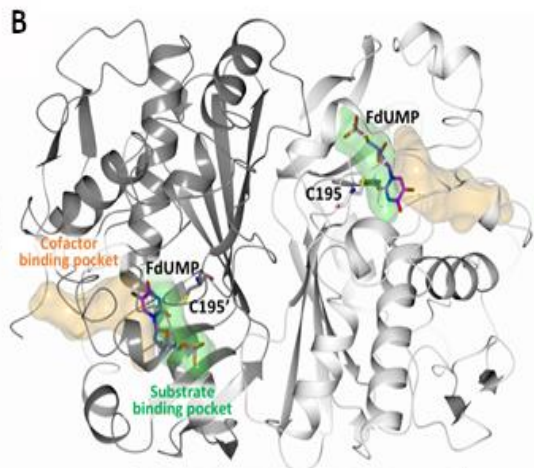

D

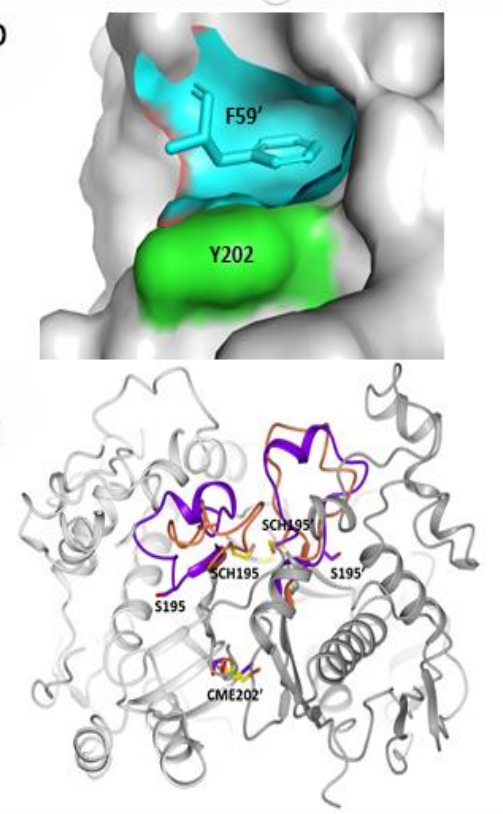

Figure 1. Drug target sites on hTS: the active-site $(A, B)$ and the Y202 pocket $(C-F)$. Drugs that are directed to the active site of hTS are able to mimic either the cofactor MTHF or the substrate dUMP and bind in their binding pockets. A, hTS (in cartoon with the A and B subunits colored white and grey, respectively) inhibited by the cofactor analogue pemetrexed (PMX, in sticks, purple carbons), which occupies the cofactor binding pocket by establishing a $\pi-\pi$ interactions with the substrate dUMP (in sticks, green carbons) (PDB ID: 1JU6). The substrate and cofactor binding sites are represented as green and orange surfaces, respectively. The catalytic cysteine, C195, is highlighted in sticks. B, The substrate analogue 2'-fluoro-2'-deoxyuridine monophosphate (FdUMP) (in sticks, purple carbons) binds inside the catalytic cavity, mainly filling the substrate binding pocket (PDB ID: 3H9K, hTS variant R163K). C, Location of F59, Y202 and CME202 residues at the hTS dimer interface by superimposition of native hTS and hTS-C195S-Y202C double mutant structures (CME: S,S(2-hydroxyethyl)cysteine; PDB-ID: 3N5G, 4O1X). D, Surface of the Y202 pocket (cyan) 
filled by F59' from the opposite monomer (cyan sticks). The surface of Y202 is highlighted in green (PDB-ID: 3N5G). E, Surface of the C202 pocket (cyan) filled by F59' from the opposite monomer (cyan sticks) in the C195S-Y202C double mutant (PDB-ID: 4O1X). The surface of C202 (oxidized as CME) is highlighted in green. F, Superimposition of the hTS-C195S-Y202C and hTS-Y202C crystal structures with the catalytic loop in hTS-Y202C (inactive conformation, in orange) and in hTS-C195S-Y202C (active conformation, in violet); notice the different orientations of the catalytic residue couples S195/SCH195 and S195'/SCH195' (PDB-ID: 4O1X, 4O1U) (SCH: S-methyl-thiocysteine). The one-letter code is used for the standard amino-acids, while for the chemically modified amino-acid, a conventional 3-letter code is used as described above.

\section{Results}

\section{Choice of the ligand binding-site on the target protein surface.}

To target the monomer-monomer interface and dissociate the hTS dimer, the 'cysteine tethering' approach to fragment-based drug design was applied $(10,11)$. We investigated the crystal structures of the active and inactive hTS (PDB-IDs 1HVY and 1YPV, respectively) and carried out molecular dynamics (MD) simulations of the monomeric and dimeric forms of the enzyme to explore the monomer-monomer interface of homodimeric hTS and identify targetable binding pockets and residues suitable to be mutated to cysteine for the tethering experiments (Supplementary Materials, Tables S1, S2). We selected the 'Y202 pocket', which accommodates the side-chain of F59' from the other monomer, as the most promising region to target (Fig. 1C-F, Fig.2A-B, Fig. S1a-b) (12). This pocket lies in the perimeter space of the intermonomer interface and is accessible in the dimeric form of the protein. It is large enough $\left(22 \AA^{3}\right)$ to accommodate a phenyl group and has several smaller crevices around it. During the MD simulations of monomeric hTS, the sidechain of Y202 moved away leaving a larger pocket and behaving like a gate. We therefore made the Y202C mutant and screened for compounds able to form a covalent disulfide bond with C202 from a library of organic disulfides. We also mutated the active site cysteine to serine to prevent the selection of compounds 
that bound at the active site (C195S). We made mutants with single point mutations at $\mathrm{C} 195 \mathrm{~S}$ and Y202C to test their functional and structural properties (Supplementary Material, Tables S1 and S2, Fig. S1) as well as the double mutant, hTS C195S-Y202C, that was used for the ligand selection according to the above described tethering approach. As expected from removal of the catalytic C195 residue, this double mutant was inactive, but X-ray crystallography showed that it takes the active conformation of hTS (13). (Fig 1F, Fig.S2, Supplementary Material, Table S3, S4). Thus, we employed the C195S-Y202C double mutant to capture compounds that, driven by some affinity for the region near residue 202, formed a covalent disulfide with C202. These compounds were selected by their stability to reducing concentrations of BME, and the C202-S-S-small-compound disulfides formed were identified by mass spectrometry (Table S6).

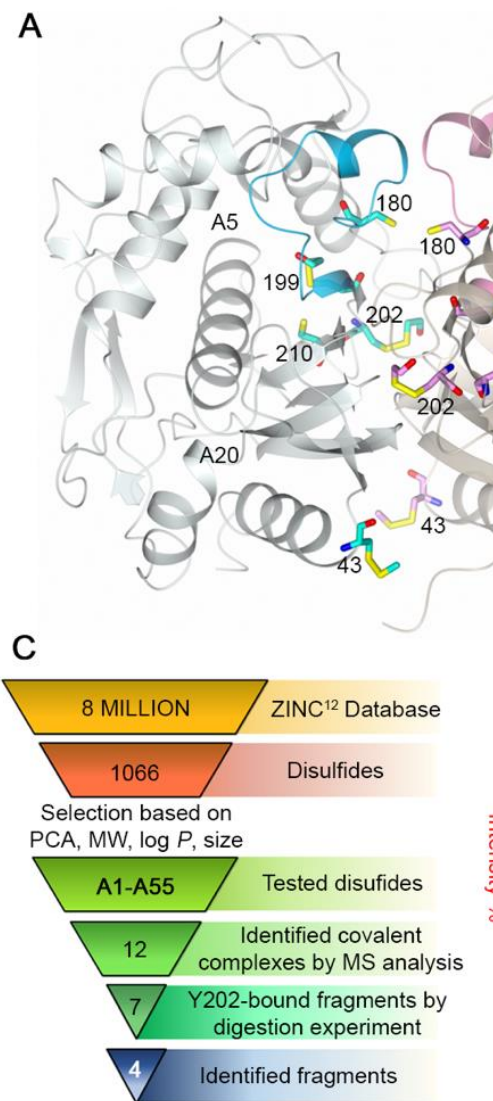

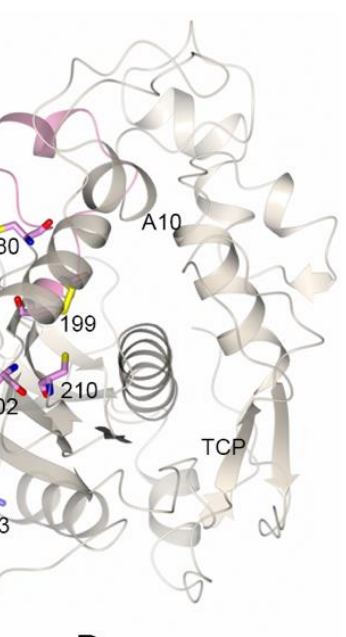

D

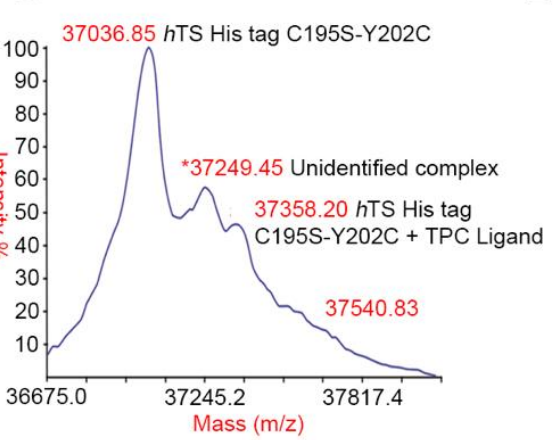

B MRGSHHHHHHGS (43)
(1) MPVAGSELPRRPLPPAAQERDAEPRPPHGELQYLGQIQHI 40 LRCGVRKDDRTGTGTLSVFGMQARYSLRDEFPLLTTKRVF 80

WKGVLEELLWFIKGSTNAKELSSKGVKIWDANGSRDFLDS 120 LGFSTREEGDLGPVYGFQWRHFGAEYRDMESDYSGQGVDQ 160 LQRVIDTIKTNPDDRRI IMCAWNPRDLPLMALPPSHALCQ 200 (180) (199) FCVVNSELSCQLYQRSGDMGLGVPFNIASYALLTYMIAHI 240 (202) (210) TGLKPGDFIHTLGDAHIYLNHIEPLKIQLQREPRPFPKLR 280 ILRKVEKIDDFKAEDFQIEGYNPHPTIKMEMAV 313

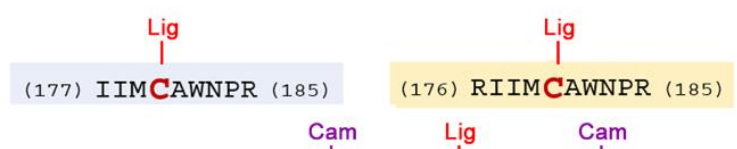

Cam Lig Cam

(186) DLPLMALPPSHALCQWNPRFCVVNSELSCQLYQR (215)

E

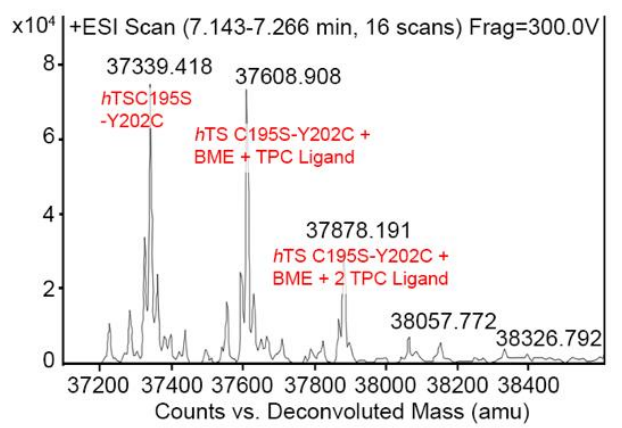

Figure 2. Disulfide library design and screening on the hTS C195S-Y202C variant. A, crystal structure of the hTS C195S-Y202C dimer (in cartoon, A and B subunits are colored pale blue and grey, respectively; the catalytic loops, in active conformation, are highlighted in cyan and pink, 
respectively) showing the positions of the cysteine residues (in sticks, carbon atoms are colored cyan and pink in subunits A and B, respectively. Compounds A5, A10, A20 and the reference compound N-tosyl-D-prolinecysteamine (TPC) were selected through the tethering experiments. They are indicated close to the peptide sequence found in the mass spectrometry identification process (Fig.2B). Their chemical structures are reported in Fig.3. B, sequence of the hTS C195S-Y202C variant and cysteine-containing peptides generated by trypsin digestion. Cysteine residues are displayed in red (the residue number is reported below each cysteine) and the mutated S195 (the catalytic residue) in blue. Three cysteine-containing peptides were generated by proteolytic cleavage of the hTS C195S-Y202C variant: peptide I177-R185 and peptide R176-R185 containing C180 and peptide D186-R215, containing C199, C202 and C210. Ligand (Lig)-bound cysteines and those alkylated as carbamidomethyl (Cam)-cysteines are displayed. C, Screening cascade from the 6 million compounds from the ZINC database to the final 4 fragments expected to bind in the Y202 pocket. D, E, Representative MS spectra in the hTS-binding evaluation step. Maldi (D) and ESI (E) MS spectra for the reference compound TPC (16). The peak on the left is the protein hTS C195SY202C; the peak in the middle is the protein bound to BME and TPC; the lefthand peak is the protein bound to BME and 2 molecules of TPC.

\section{Mass-spectrometric ligand selection.}

To identify molecules able to bind near Y202, we designed a library of commercially available compounds from the ZINC database (14). (Fig.2C). We first selected 1066 disulfide compounds, that were either symmetric (RSSR) or asymmetric $\left(\mathrm{R}_{1} \mathrm{SSR}_{2}\right)$, with drug-like or fragment-like properties. Thirteen out of the 55 compounds selected for testing were found to bind the protein according to the results of both MS techniques (Fig. 2D, 2E, Supplementary Material, Table S5 and S6). Among them, 7 compounds, A5, A6, A10, A15, A20, A38 and TPC, were selected based on the feasibility of their chemical modification and were further analyzed by a bottom-up MALDI-TOF and ESI-QTOF MS proteomic analysis to identify the cysteine residue they had bound (Table S7). The 7 compounds 
bound either peptides (I177-R185) IIMCAWNPR (sequence A) or (R176-R185) RIIMCAWNPR (sequence B), both containing the $\mathrm{C} 180$ residue of the digested protein (Table S7). Four molecules, A5, A10, A20 and TPC also bound to peptide (D186-C215) DLPLMALPPSHALCQFCVVNSELSCQLYQR (sequence C) (Table S7), containing the engineered Y202C together with the native C199 and C210. In agreement with the described computational and crystallographic data (see above), Y202C is the most exposed of these three cysteines and is thus easily available for disulfide-bond formation with the RS fragments, being therefore suitable for tethering experiments. Other reasons to select the Y202 pocket are that Y202 is not predicted to be part of the mRNA binding region and has the capability to interact with various functional groups. Hence, despite the difficulties in the identification of the precise cysteine to which the fragments could bind, all the above arguments support the choice of the Y202 pocket at the hTS interface as a suitable binding site for further structure-based studies. Therefore, we considered the fragments A5, A10, A20 and TPC for further medicinal chemistry modifications (Fig.3A).

A

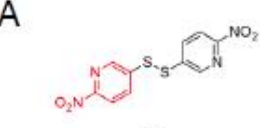

A5

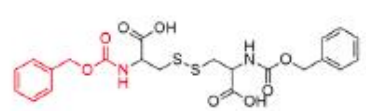

A20

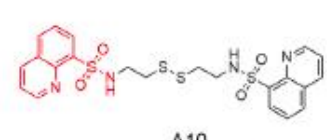

A 10

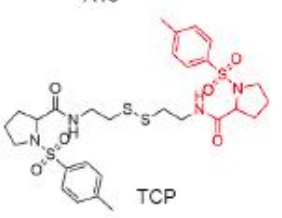

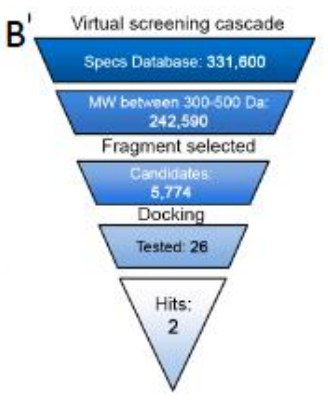

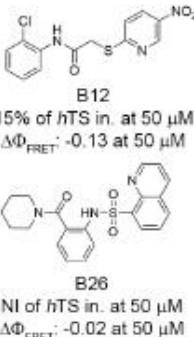

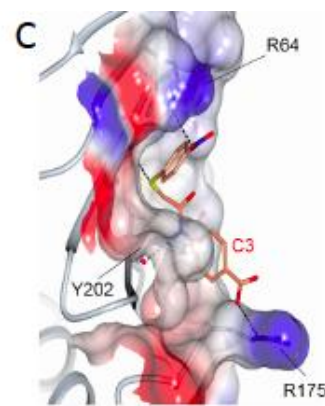

D

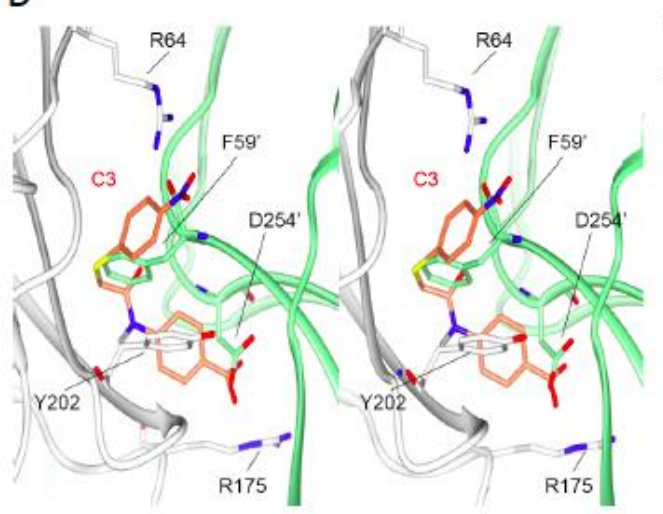

E

Structural changes and associated biological properties

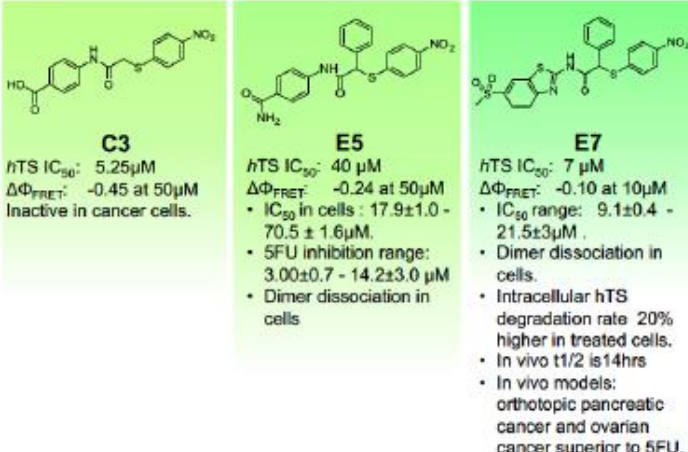


Figure 3. From hits to dimer-disrupting compounds. A, Structures of the compounds identified in MS studies (in red the fragments used for the virtual screening approach to the design of the compound library without the disulfide bonds). B, Virtual screening cascade and chemical structures of the identified compounds, B12 and B26. C, Detail of the binding mode of compound $\mathbf{C 3}$ (orange sticks) with the hTS interface residues R64, Y202 and R175 (positive and negative potential regions are colored in blue and red, respectively) obtained with docking methods. D, Stereo view of the superimposition of the calculated binding mode of $\mathbf{C 3}$ onto the first monomer (white cartoon) with the opposite monomer overlapping (lime cartoon). Atom color code: nitrogen - blue; oxygen - red; sulphur - yellow. E, Structural modification of compound B12. The changes in chemical structure and the associated $\Delta \Phi_{\mathrm{FRET}}$, hTS dimer dissociation in cells, cellular growth inhibition and in vivo data of the key compounds $\mathbf{C 3}, \mathbf{E 5}$ and $\mathbf{E 7}$ of the sub-series are reported. 


\section{From low-affinity compounds to TS homodimer disrupters.}

Starting from the four fragments identified by tethering/MS (TPC, A5, A10, A20) (Fig.3A), we performed an analogue search that yielded a dataset of 331,600 commercially available compounds (Specs dataset, www.specs.net) potentially able to bind at the Y202 pocket of the monomer/monomer interface. The initial dataset was progressively reduced to 5,774 candidates using MW, structural and chemical criteria (Fig. 3B). These were then docked into the Y202 pocket with the software FLAP (15) using a receptor-based pharmacophore model built from the X-ray crystal structure of hTS (PDB: 1HVY). A final set of 26 molecules (B1-B26, Table S8) was selected to be tested for their ability to inhibit hTS and destabilize its dimeric form by, respectively, kinetic and FRET (Förster resonance energy transfer) assays (16). The latter had been previously established to investigate the dimer/monomer equilibrium of hTS and measure the corresponding dissociation constant (2). In this experiment, we conjugate one protein monomer with red-emitting tetramethylrhodamine $(\mathrm{T})$ and the other with green-emitting fluorescein $(\mathrm{F})$. Only when the monomers are close to each other, i.e. in the dimeric assembly, does F-to-T excitation energy transfer occur, a FRET signal is detected and its efficiency is determined. Thus, this assay affords a direct evaluation of the effect of the compounds on the dimer/monomer equilibrium of hTS. Among the 26 compounds selected by VS, B12 and B26 (Fig. 3B, Table S8), caused low but reproducible decreases in the efficiency of FRET $\left(\Delta \Phi_{\text {FRET }}=-0.13\right.$ and $\Delta \Phi_{\text {FRET }}=-0.02$, respectively), thus indicating some ability to perturb the enzyme dimer. In the docked poses, interactions were established by B12 with Y202 through the $o$-chloro-phenyl ring and with R175 through the p-nitro-pyridyl ring, and by B26 with Y202 through the piperidin-1-yl ring and with R175 through the quinoline ring (Fig.3C, D). Next, we designed and synthesized 4 sets of compounds (C-F) to develop B26 into a biologically active inhibitor able to disrupt the dimer and 
to show inhibition of recombinant hTS (Supplementary Materials, Figs.S3-S9 and Tables S9-S12). The medicinal chemistry strategy led to compounds $\mathbf{C 2}, \mathbf{C 3}$ (Fig.3) and $\mathbf{C 4}$ that featured $\mathrm{IC}_{50}$ values between $5.25 \mu \mathrm{M}$ and $246 \mu \mathrm{M}$ (Table S9) and were structurally different only for the position of the carboxylic group on the phenyl ring. These compounds were selected for detailed mechanistic recombinant hTS studies and FRET analysis (see "Mechanistic analysis of dissociative inhibition.” Section below). Finally, the most interesting compounds for our study were E5, E6 and $\mathbf{E 7}$ with $\mathrm{IC}_{50}$ values against hTS of, respectively, 40, 10 and $7 \mu \mathrm{M}$ and decreased FRET efficiency by -0.24 at $50 \mu \mathrm{M}(\mathbf{E 5}, \mathbf{E 6})$ and -0.1 at $10 \mu \mathrm{M}$ (E7, Fig.3E). The compound that was most active on cells (vide infra), E7, was determined to be a racemic mixture of two enantiomers that equilibrated with a half-life of $56 \mathrm{~min}$ (see details in 'Enantioseparation and racemization of E7' in the Supplementary Material, Table S13, Figs. S14-S15).

\section{Mechanistic analysis of dissociative inhibition.}

Compounds $\mathbf{C 3}$ (Fig.3E) a nitrothiophenyl derivative and its isomers, C2, C4 (Table S9), are structurally similar to compound E7 (Fig.3E) and are much more soluble in water. So they were used for quantitatively investigating the molecular mechanistic basis of their ability to both inhibit recombinant hTS and disrupt its dimer. The three isomeric compounds showed different inhibitory potencies against $300 \mathrm{nM} \mathrm{hTS}$, with $\mathrm{IC}_{50}$ values of $83,5.25$ and $246 \mu \mathrm{M}$, respectively. Consistently, $\mathbf{C 3}$ caused the largest decrease in FRET efficiency $\left(\Delta \Phi_{\text {FRET }}\right)$ at concentrations lower than $50 \mu \mathrm{M}$

(Fig. 4A, 4B). However, because an observed $\Delta \Phi_{\text {FRET }}$ might result from the ligand causing a structural distortion yielding a spatial F/T arrangement less favorable for energy transfer, rather than true dissociation of the enzyme dimer, we searched for clear evidence of the disruptive character of this inhibitor by investigating the dependence of the dose-effect curves on total protein 
concentration, $\mathrm{E}_{\mathrm{T}}$ (Fig. 4C). At each inhibitor concentration, as well as at saturation, $\Phi_{\mathrm{FRET}}$ decreased with $\mathrm{E}_{\mathrm{T}}$ according to a dimer-monomer equilibrium model (2) (Fig. 4D). As expected for a dissociative inhibition mechanism, the results were consistent with a 5 -fold increase in the equilibrium dissociation constant for the protein bound to $\mathbf{C 3}$ with respect to the free protein. This change, and the corresponding decrease in $\Delta \mathrm{G}^{\circ}$ for dimer disruption, $\Delta \Delta \mathrm{G}^{\circ}=-4 \mathrm{~kJ} \mathrm{~mol}^{-1}$, are direct evidence of the dimer-destabilizing ability of compound $\mathbf{C 3}$.

With the aim of extracting quantitative mechanistic data from the dose dependencies of the inhibition and the $\Phi_{\text {FRET }}$ data, we employed the equilibrium and kinetic scheme shown in Fig. 4E. This was analyzed as reported in the Supplementary Material (Analysis of combined equilibria and fitting to FRET data) with the aim of relating the observed $\Phi_{\text {FRET, that in our conditions is a }}$ direct measure of the mole fraction of hTS dimers, to the enzyme and inhibitor concentrations. The $\Phi_{\text {FRET }}$ data in panel 4B were fitted to Eq. S3 in the Supplementary Material to obtain the reported equilibrium parameters for compounds C2-C4. From these, we conclude that, when saturating hTS, the three compounds have similar effects on the dimer stability: they cause a decrease in $\Delta \mathrm{G}^{\circ}$ for dimer dissociation from -40.5 to about $-36.5 \mathrm{~kJ} \mathrm{~mol}^{-1}$. On the other hand, their different abilities to decrease the FRET efficiency, i.e. to induce dimer dissociation, at lower concentrations is due to different affinities for the protein dimers, with $\mathrm{K}_{\mathrm{I}}$ "s of $2.8 \times 10^{-5}, 1 \times 10^{-5}$ and $2 \times 10^{-5} \mathrm{M}$, for $\mathbf{C 2}, \mathbf{C 3}$ and $\mathbf{C 4}$, respectively. Thus, at concentrations far from enzyme saturation by these inhibitors, the observed overall effect results from a combination of the inhibitor's affinity for the protein and its ability to destabilize the dimeric assembly by impairing crucial attractive intermonomer interactions.

Binding of compounds C2-C4 at the Y202 pocket of the hTS monomer/monomer interface is supported by computational docking (Fig. 3C, D). In the hTS dimer, the aromatic ring of the Y202 
residue forms a $\pi-\pi$ interaction with the phenyl ring of the F59' residue from the opposite subunit. Such a ring is replaced by the phenyl ring of the benzoic acid portions of C2-C4 in their modeled complexes with hTS. Thus, these compounds mimic some of the residue side chains from the opposite monomer in the dimer, providing similar interactions in this region. This finding is supported by the higher affinities of the inhibitors for the hTS monomer $\left(\mathrm{K}_{\mathrm{I}}=10^{-6} \mathrm{M}\right)$ than for the dimer $\left(\mathrm{K}_{\mathrm{I}}{ }^{\prime}=1.7 \times 10^{-5} \mathrm{M}\right)$, as obtained by fitting of the FRET data in Fig. 4B: the gain in free energy associated with binding of the inhibitor to the free monomer is partially compensated when it binds the dimer by the disruption of the above-mentioned interactions between the residues from the two monomers.

The reliability of our docking virtual experiments was strengthened by their ability to explain the observed higher affinity for hTS of compound $\mathbf{C 3}$ relative to its isomers, $\mathbf{C 2}$ and $\mathbf{C 4}$. While the nitro-groups of all these ligands can make head-to-head hydrogen-bonding interactions with R64 (Fig. 3C-D), only the para carboxylic group of compound $\mathbf{C 3}$ arranges properly to establish an additional H-bond with R175. Instead, the meta and ortho carboxylic groups of $\mathbf{C} 2$ and $\mathbf{C} 4$ do not have the correct orientations for interacting with this arginine residue. 
A

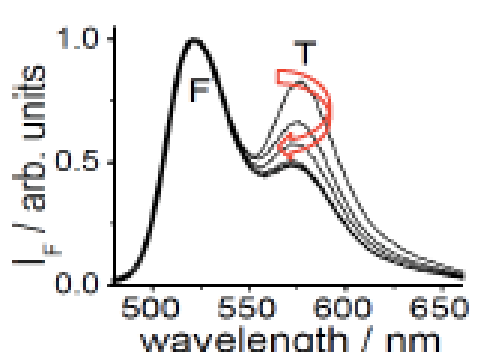

C

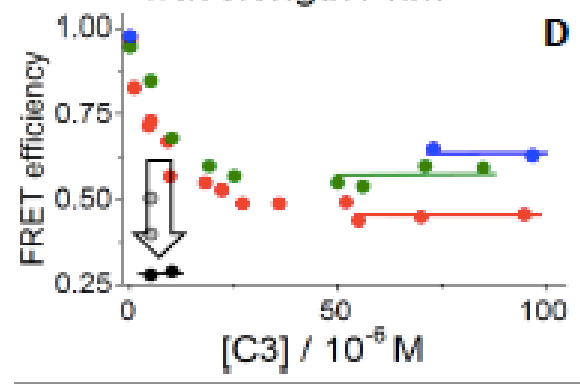

E
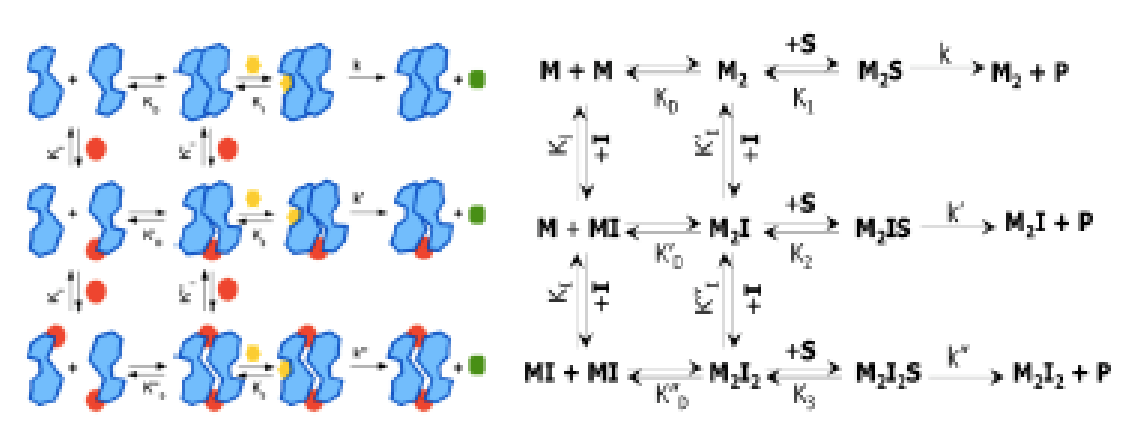

F

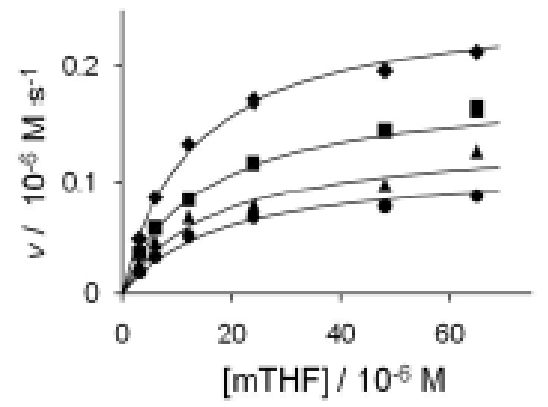

Figure 4. Spectroscopic and mechanistic analysis of hTS dissociative inhibition for compounds C2, C3 and C4. A, Emission spectra of fluorescein(F)- and tetramethylrhodamine(T)labeled hTS $\left(\lambda_{\text {exc }}=450 \mathrm{~nm}\right)$ : the decreasing emission contribution of T relative to $\mathrm{F}$ with increasing concentrations of $\mathbf{C 3}(0,4.5,9.5,15,25,100 \mu \mathrm{M})$ parallels the decrease in the hTS dimer mole fraction. B, Dependence of the observed FRET efficiency on the concentrations of three inhibitors 
(C3, black; C4, blue; C2, red); the hTS dimer concentration was $100 \mathrm{nM}$. The lines represent the best fittings of the experimental results to Equation S3 (Supplementary Materials). The most significant fitting parameters are reported. C, Dependence of FRET efficiency on the concentration of $\mathbf{C 3}$ with total protein concentrations of $490 \mathrm{nM}$ (blue circles), $250 \mathrm{nM}$ (green circles), $100 \mathrm{nM}$ (red circles) and $50 \mathrm{nM}$ (black circles). The horizontal segments represent the values of the efficiency in the limit of high inhibitor concentration. At $\mathrm{E}_{\mathrm{T}}=50 \mathrm{nM}$ and $\mathrm{I}_{\mathrm{T}}=5 \mu \mathrm{M}$ the vertical arrow indicates the evolution of the measured value of the FRET efficiency 5, 7 and 12 minutes after inhibitor addition (grey and black circles). D, Analysis of the hTS monomer-dimer equilibrium according to eq. $\Phi_{\mathrm{FRET}}=1-0.5\left(\Phi_{\mathrm{FRET}} / \mathrm{E}_{\mathrm{T}}\right)^{1 / 2} \mathrm{~K}^{1 / 2}$ (see ref. 7 ) and corresponding equilibrium constants without $\mathbf{C 3}$ (open circles, $\mathrm{K}=\mathrm{K}_{\mathrm{D}}$ ) and at saturating $\mathbf{C 3}$ concentrations (closed circles, $\mathrm{K}=\mathrm{K}{ }^{\prime}{ }_{\mathrm{D}}, \Phi_{\mathrm{FRET}}=$ FRET efficiency). E, Cartoon and standard representations of the dissociative inhibition mechanism of hTS. Blue shapes represent enzyme monomers (M) in the active conformation bound to dUMP; red dots indicate a dissociative inhibitor (I), yellow hexagons, the folate substrate $(\mathrm{S})$, green octagons, the product. F, Dependence of the initial reaction rate $(v)$ on mTHF concentration (free substrate, $[\mathrm{S}]$, and total, $\mathrm{S}_{\mathrm{T}}$ ) at four different $\mathbf{C 3}$ concentrations (from top to bottom, $[\mathrm{I}]=0,12,24$ and $36 \mu \mathrm{M}$ ). The fitting curves represent equation $v=\mathrm{x}_{\mathrm{r}}^{2} \mathrm{~S}_{\mathrm{T}} \mathrm{D}$ derived in the 'Analysis of a dissociative inhibition mechanism and fitting to data' of the Supplementary Information and computed with $\mathrm{E}_{\mathrm{T}}$ (total enzyme dimer concentration) $=300$ $\mathrm{nM}, \mathrm{k}=\mathrm{k}^{\prime}=0.9 \mathrm{~s}^{-1}, \mathrm{k}^{\prime}{ }^{\prime}=0.1 \mathrm{~s}^{-1}, \mathrm{~K}_{1}=\mathrm{K}_{2}=10^{-5} \mathrm{M}, \mathrm{K}_{3}=2 \times 10^{-5} \mathrm{M}, \mathrm{K}_{\mathrm{I}}=10-6 \mathrm{M}, \mathrm{K}_{\mathrm{I}}{ }^{\prime}=\mathrm{K}_{\mathrm{I}}{ }^{\prime}=10^{-5} \mathrm{M} . \mathrm{x}=$ $\left[\mathrm{M}_{2}\right]^{1 / 2}$ and using for $\mathrm{x}_{\mathrm{r}}$ the only acceptable root of the quadratic equation in panel $4 \mathrm{f}$.

As for the mechanism of inhibition of hTS enzyme activity by compounds C2-C4, we show that the scheme in Fig. 4E can account for the dependence of the reaction rate $(v)$ on the substrate 
concentration at different inhibitor concentrations with very reasonable fitting parameters (Fig. 4F). Simple observation of the dependence of $v$ on [C3] with total enzyme concentration ( $\left.\mathrm{E}_{\mathrm{T}}\right) 300$ $\mathrm{nM}$ (Fig. S10), indicates a regular decrease with increasing [C3] to a limiting rate that is about 1/5 the rate in the absence of inhibitor. On the other hand, the $\mathrm{E}_{\mathrm{T}}$-dependent limiting value of $\Phi_{\mathrm{FRET}}$ was about half the full one (Fig. 4B). This difference in the limit behaviors of $v$ and $\Phi_{\text {FRET suggests }}$ that, when bound to the inhibitor, the dimeric enzyme is less catalytically active than when free. Or, in terms of the dissociative/noncompetitive model in Fig. $4 \mathrm{E}, \mathrm{K}_{2}$ and $\mathrm{K}_{3}$ are larger than $\mathrm{K}_{1}$, and/or k' and k" are smaller than k. The curves that go through the kinetic data in Fig. $4 \mathbf{F}$ are plots of eq. $v=\mathrm{x}_{\mathrm{r}}^{2} \mathrm{~S}_{\mathrm{T}} \mathrm{D}$ that was obtained by fast-equilibrium solution of the scheme in Fig. 4E (for the derivation of the equation and the meanings of the symbols therein, see the Analysis of a dissociative inhibition mechanism and fitting to data in the Supplementary Material). The fitting parameters, provided in the caption, are consistent with the results of the FRET-based analysis of the equilibria in Fig. 4E and the $v$-vs- $\Phi_{\text {FRET }}$ limit behavior. In detail: i) $\mathrm{k}$ " is much smaller than $\mathrm{k}$ and $k$ ', i.e., $\mathrm{M}_{2} \mathrm{I}_{2}$ is almost catalytically inactive, a feature typical of noncompetitive inhibition; ii) $\mathrm{K}_{3}$ is larger than $\mathrm{K}_{1,2}$, i.e., inhibitor binding reduces the enzyme affinity for MTHF; iii) $\mathrm{K}_{\mathrm{I}}{ }^{\prime}, \mathrm{K}_{\mathrm{I}}{ }^{\prime}$, remain one order of magnitude larger than $\mathrm{K}_{\mathrm{I}}$, i.e., when saturated with dUMP, the enzyme dimer shows lower affinity for the inhibitor than the monomer; iv) using the relationships among the equilibrium constants, we estimate that $\mathrm{K}_{\mathrm{D}}{ }^{\prime} / \mathrm{K}_{\mathrm{D}}=\mathrm{K}_{\mathrm{I}}{ }^{\prime} / \mathrm{K}_{\mathrm{I}} \approx 10$ and $\mathrm{K}_{\mathrm{D}}{ }^{\prime}{ }^{\prime} / \mathrm{K}_{\mathrm{D}}{ }^{\prime}=\mathrm{K}_{\mathrm{I}}{ }^{\prime}{ }^{\prime} / \mathrm{K}_{\mathrm{I}} \approx 10$, i.e., the kinetic results confirm a progressive dissociation of the enzyme dimer to monomers upon subsequent addition of $\mathbf{C 3}$ (Analysis of a dissociative inhibition mechanism and fitting to data in Supplementary Material). The latter is therefore confirmed to be both a dimer disrupter and a dissociative inhibitor, the two roles being intimately connected with each other. 


\section{The dimer disrupter engages hTS in cancer cells.}

To gain evidence that our hTS dimer disrupters engage this enzyme in cells, we performed fluorescence microscopy. For this experiment, we selected compound $\mathbf{E 5}$ to be incorporated in the final conjugated probe because its chemical precursor, E3, shows a reactive carboxyilic group (Table S11, Fig.3E) and therefore suitable for probe conjugation chemistry. E6 and E7 did not present the same suitable precursors. Using a short ether linker, we conjugated it to fluorescein isothiocyanate (FITC), a green-emitting probe that favors cell internalization (for details see Supplementary Material and Fig.S11-S15). The E5-(O) $\left.\mathbf{O}_{2} \mathbf{O c}\right)$-CAM-FITC (E5-FITC) conjugate is permeable to the cells though it proved slightly less active at inhibiting recombinant hTS than $\mathbf{E 5}$ $\left(\mathrm{IC}_{50 \mathrm{~S}}=40\right.$ and $100 \mu \mathrm{M}$, for $\mathbf{E 5}$ and $\mathbf{E 5 - F I T C}$, respectively), likely because the probe tail caused some steric hindrance for binding to the enzyme. We ectopically expressed in HT-29 cells either Wild Type (WT) hTS or a mutant with a tetracysteine motif (CCPGCC, TC) inserted near the Nterminus (TS-TC) (16). The tetracysteine motif tightly binds ReAsH, a rhodamine-based diarsenical probe. The ReAsH/TC-TS complex emits red fluorescence with much higher efficiency than the unbound probe. HT-29 cells (transfected with either WT TS or TC-TS) were treated with ReAsH and E5-FITC. Fluorescein transferred excitation energy to rhodamine in the ReAsH-TChTS/E5-FITC complex, thus signaling its formation with a FRET signal. Indeed, our image analysis (Fig.5) showed that cells expressing hTS-TC produced a significantly higher $(\mathrm{P}<0.0001)$ average FRET signal (4222 \pm 2128$)$ than control cells that expressed WT hTS $(1250 \pm 1178)$, thus proving the occurrence of intracellular hTS/E5 engagement. 


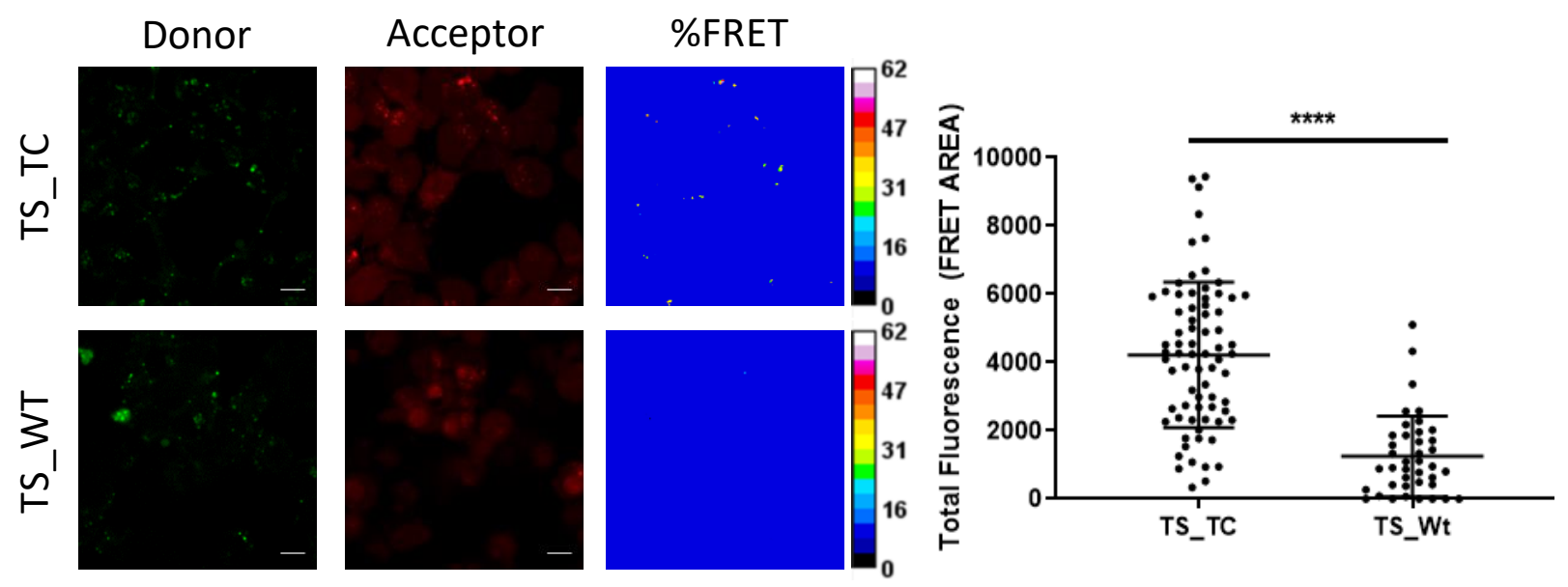

Figure 5. Microscopic analysis of hTS dissociative inhibition. Representative fluorescence microscopy images of TS-TC- and WT-TS-expressing HT-29 cells stained with red-emitting ReAsH and incubated with green-emitting E5 -FITC; from left to right: green-emission and redemission channels, and FRET efficiencies depicted according to the colour scale (\%) in the right bars. Right: comparison of the average FRET areas for cells overexpressing TS with (TS_TC) or without (TS_Wt) ReAsH binding sites, obtained using PixFRET within ImageJ. Each dot represents the total FRET area of an individual field with at least 10 cells. The statistical analysis was performed using unpaired two tailed t-test $(\mathrm{P}$ value $>0,0001)$ using Prism 8 for windows (version 3.1.1).

\section{Cancer cell growth inhibition by hTS dimer disrupters.}

The compounds that both inhibited recombinant hTS and decreased the FRET efficiency, i.e., C2C5, C9, C10, C13, D5-D9, D12, E1 and E3-E7 were selected to test cancer-cell growth inhibition. They were tested as racemates. We employed 5-FU, metabolized to FdUMP inside cells, as a reference in these experiments. Tests were performed on several model cancer cell lines. Because 5-FU is a key compound in the pancreatic-cancer first-line regimen FOLFIRINOX, we chose two pancreatic adenocarcinoma primary cell cultures, PDAC 2 and PDAC 5, which express low and 
high TS levels, respectively (17). These cultures were obtained from radically-resected patients which reflected the histopathological and genomic features of human PDACs (23). Other models used in our investigation were two ovarian cancer cell lines, IGROV1 and TOV112D, and two gynecological cancer cell lines that feature high hTS levels resulting from cross-resistance to cisplatin $(\mathrm{C13} *$ A2780/CP) as well as the corresponding cisplatin-sensitive lines (2008, A2780) (9). Since 5-FU is always included in the treatment of colorectal cancer, we added three epithelial colorectal cancer cell lines, HCT116, HT29 and LoVo, which exhibit different sensitivities to 5FU (18). We observed similar results in all tested cell lines. While most tested compounds produced weak effects (Fig S16, Table S14), for the compounds E5-E7 we were able to determine $\mathrm{IC}_{50}$ values (Fig.6A, Fig. S16, S17, Table S15). E7 was the most interesting among these compounds, being active on all the cancer cells tested with $\mathrm{IC}_{50}$ values between 10 and $37 \mu \mathrm{M}$ after 72 hours of exposure, therefore showing a lower or comparable activity, in a few cases, with respect to 5-FU.

A crucial issue remains the intracellular mechanism of action of the dimer disrupters. Among the classical active-site inhibitors of hTS, 5-FU forms a stable ternary complex with the MTHF cofactor and the hTS protein and induces high expression of hTS by stabilizing the dimeric assembly, thereby impairing both the protein feed-back regulation and the proteasomal degradation $(18,19,20,21)$. On the other hand, PMX, another TS active-site inhibitor with a folate-analog structure, binds at the MTHF binding site and forms a reversible non-covalent ternary complex with dUMP $(3,22)$ We have shown that our dimer disrupters inhibit recombinant hTS with a different mechanism of action; thus, we expect them to act differently in cells too, and to produce a different modulation of the intracellular hTS levels with respect to 5-FU and PMX. 


\section{The dimer disrupters decrease the hTS levels and promote cell death.}

To investigate the intracellular mechanism of action of compounds $\mathbf{E} \mathbf{5}$ and $\mathbf{E 7}$, we first determined whether they induce apoptotic cell death both in cisplatin-sensitive A2780 cells and in -resistant A2780/CP cells. As a control, we included cisplatin that, as expected, caused a higher rate of apoptotic cell death in sensitive than in resistant cells (Fig. S18 a,b, Fig. S19). Remarkably, also E7 induced apoptotic death in the two cell lines after a 48 hours treatment with a slightly higher efficiency than PMX (Fig. 6B, C).
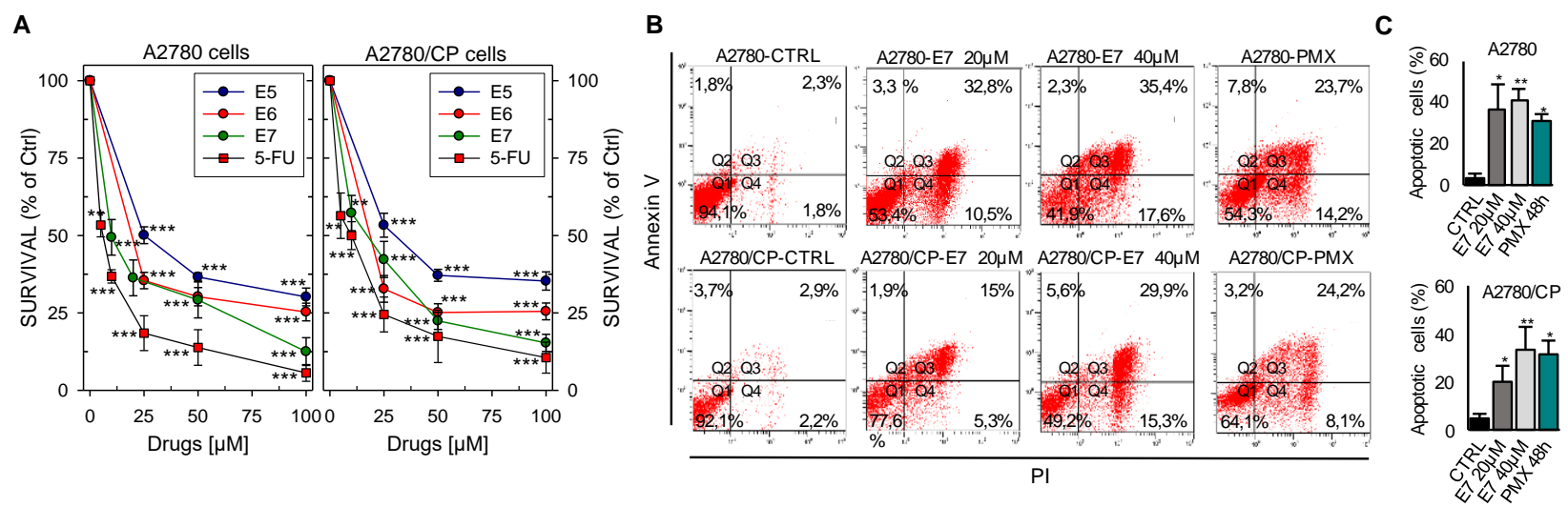

Figure 6. Effects of hTS inhibitors in human cancer cell lines. A, dose-response curves for E5E7 and 5-FU against A2780 and A2780/CP cell growth. B, flow cytometric analysis of apoptosis of A2780 and A2780/CP cells treated with E7 or PMX (Annexin V-/PI-: live cells, Annexin V+/PI-: early apoptotic cells, Annexin V+/PI+: late apoptotic cells, Annexin V-/PI+: necrotic cells). C, quantification of Annexin V-positive cells (Q2 + Q3) was performed using Annexin V/PI kit.

Following treatment of both A2780 and A2780/CP cells with E5 and E7, we found no change in the levels of the DHFR protein, but the level of the hTS protein decreased significantly in a dose- 
dependent manner (Fig. 7A), being low to undetectable after 12-24 hrs of exposures to E7 in HT29 cell (Fig. S18c) and undetectable after 24-36 hrs of treatment in A2780/CP cells (Fig. S18d). In the same cell lines we observed increased levels of hTS after treatment with both PMX (Fig. 7B, right) and 5-FU (Fig. 7B, left).

The ubiquitin-independent proteasomal degradation pathway is an important mechanism to destroy destabilized proteins in cells, and hTS is one of the substrates of this pathway (21). The observed decrease in hTS protein levels caused by $\mathbf{E 5}$ and $\mathbf{E 7}$ led us to hypothesize that their effect might be associated with a post-translational regulation mechanism, including proteasomal degradation. To test this hypothesis, we selected E7, our best cellular inhibitor, to determine the hTS half-life after blocking the de novo protein synthesis with the cycloheximide (CHX) inhibitor. As shown in Fig. 7C, we found that the half-life of hTS was 3 hours in untreated cancer cells and that the degradation rate of the enzyme increased by $20 \%$ in E7-treated A2780 cell (Fig. 7D).

To investigate whether the $26 \mathrm{~S}$ proteasome was involved in such an accelerated degradation, we added the proteasome inhibitor MG132 (10 $\mu \mathrm{M}$, for 5 hrs) to E7-treated and untreated A2780 cells. Indeed, as shown in Fig. 7E, MG132 restored the hTS levels in treated cells. These findings are opposite to the known effects on the hTS intracellular life exhibited by the reference inhibitor, 5 FU. In fact, previous reports on other cell models have shown that $\mathbf{5 - F U}$ causes a remarkable slowdown of the TS degradation rate because most of the protein is engaged in a ternary complex with the folate substrate and the inhibitor is protected from the proteasome (20-24). To investigate whether the engagement of E5 and E7 with hTS (Fig. 5) in cancer cells could affect the protein catalytic activity, their effect on endogenous TS activity was evaluated on cellular extracts from untreated cells of four cell lines: A2780, A2780/CP, 2008 and C13*. These extracts were incubated with the inhibitors at their $\mathrm{IC}_{50}$ values for $60 \mathrm{~min}$. In these conditions, the catalytic activity of TS 
was reduced to $25-30 \%$ in the cellular extracts of all cell lines (Fig. S18 e,f). This reduction of the hTS activity supports the hTS target engagement by the dimer disruptors and link the observed effect with the dimer to monomer equilibrium shift observed in Fig. 7F (see next section).

Based on the above data, we conclude that $\mathbf{E 7}$ reduces the hTS levels in cancer cells by enhancing its proteasomal degradation. Because E7 behaves as a disrupter of the dimeric recombinant protein, we anticipate that the promotion of its proteasomal degradation in cells is due to an increased fraction of more labile monomers.

\section{E7 disrupts endogenous hTS dimers yielding monomers that are more rapidly degraded by}

\section{the proteasome.}

We then explored how treatment with E7 perturbed the intracellular hTS dimer/monomer equilibrium using a cross-linking strategy on endogenous hTS from A2780 cells (Fig. 7F) (25). These cells were treated with E7 or with the vehicle (DMSO) for 3 hrs, after which cross-linking was carried out by adding bis(sulfosuccinimidyl)suberate $\left(\mathrm{BS}^{3}\right)$ to cell lysate. Next, the hTS dimers and monomers were detected via immunoblot after SDS-PAGE in reducing conditions. The treatment caused a significant decrease of the dimer/monomer ratio relative to the control $(-40 \pm 10$ $\%$, Fig. 7F). We thus confirmed that E7 disrupts the hTS dimers stoichiometrically also in cells. To test if the hTS monomer is less stable than the dimer, we used a model system in which HCT116 cells ectopically express Myc-DDK-tagged wild type hTS (WT), or the hTS F59A dimer interface mutant. F59A variant is essentially monomeric at all physiological concentrations showing a Kd of $1.3 \times 10^{-4} \mathrm{M}$ with respect to the WT hTS ( $\mathrm{Kd}$ of $\left.10^{-8} \mathrm{M}\right)$ that is, instead, essentially dimeric (13). We analyzed the protein turnover of WT hTS and of the monomeric F59A variant, in the presence of the de novo protein synthesis inhibitor CHX. The expressions of the mRNA of the two proteins 
showed no statistically significant differences (see Method section, Fig. S20). The F59A variant was already hardly discernible at time 0 (24 hrs after ectopic expression) while the dimeric WT hTS showed the turnover of about 8.5 hours (Fig. 7G). Thus, the monomeric hTS F59A variant is unstable and undergoes much faster turnover than the dimeric WT protein (26).

A $\mathrm{A} 2780$ A2780/CP $\mathrm{A} 2780 \quad \underline{\mathrm{A} 2780 / \mathrm{CP}}$

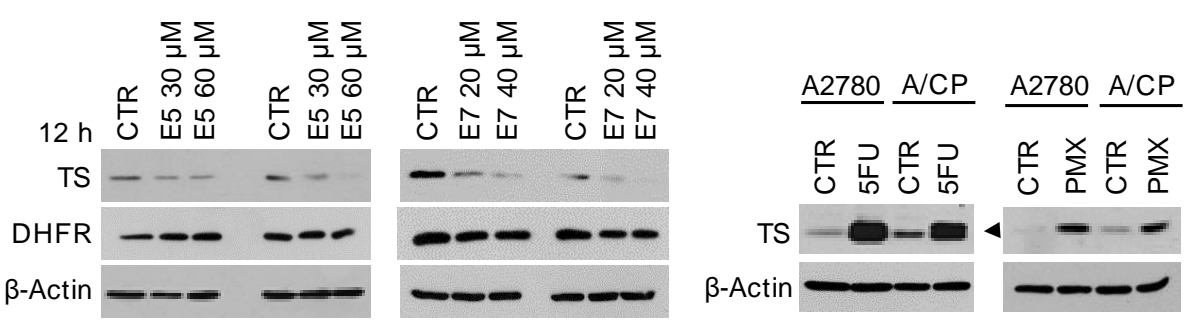

C

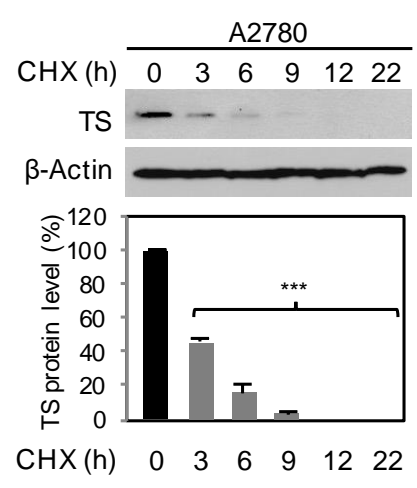

E

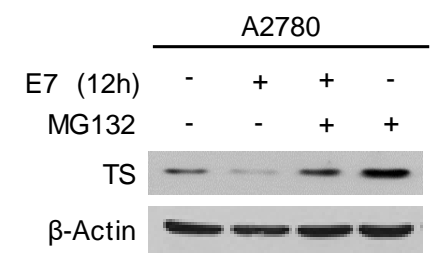

$\mathbf{F}$

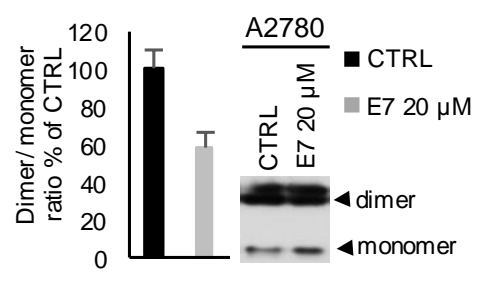

B

D

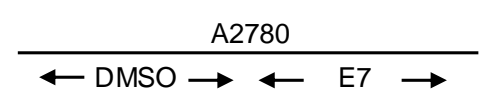

$\mathrm{CHX}$ (h) $\quad 0 \begin{array}{llllllllll} & 0 & 2 & 3 & 0 & 1 & 2 & 3\end{array}$

TS - - -

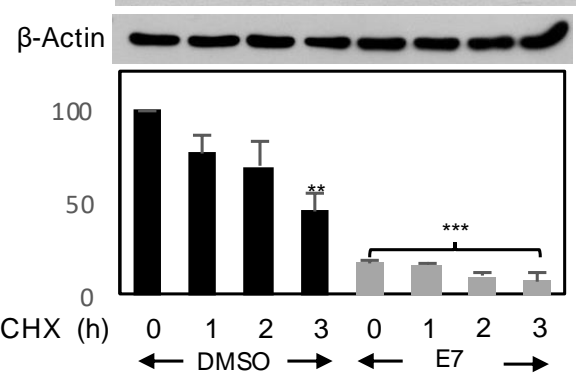

G
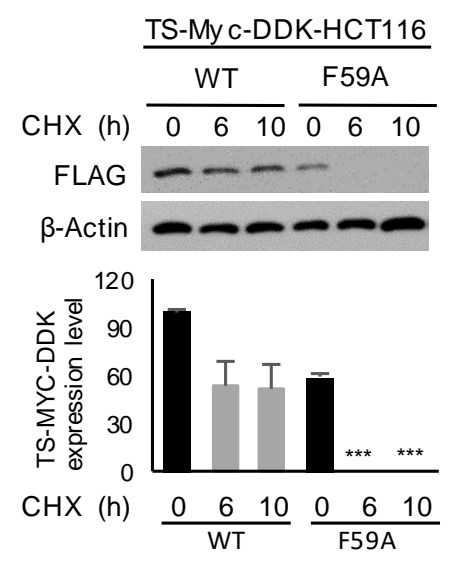
Figure 7. The dimer disrupters effect on hTS levels in human cancer cell lines. A, effects of E5 and E7 on hTS and DHFR protein levels in A2780 and A2780/CP cells. B, increase of hTS protein level in A2780 and A2780/CP cells following treatment with 5-FU (5 $\mu \mathrm{M}, 72 \mathrm{~h})$ (ternary-

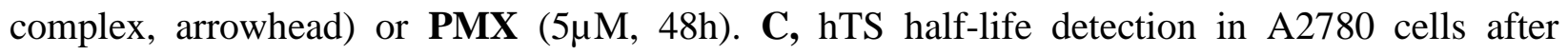
treatment with CHX (90 $\mu \mathrm{g} / \mathrm{ml})$ for 0-22h. D, stability of hTS in A2780 cells treated with E7 for 12h, followed by treatment with CHX for the indicated times. E, A2780 cells were treated with E7 for 12h, then with MG132 for 5h, $10 \mu \mathrm{M}$. F, effects of $\mathbf{E} 7$ on dimer/monomer equilibrium after 3 hours of exposure in A2780 cells. G, half-life detection of exogenous hTS protein level (antiFLAG stain) in HCT116 cells transfected with TS-Myc-DDK (CHX, 0, 6 and 10h) or F59A mutant (CHX 0, 6 and 10h) tagged vector. Data indicate mean values and standard deviation of biological repeats performed in duplicate. $\mathrm{P}$ values were calculated with two-sided Student's $t$-test and ANOVA followed by the Tukey's multiple comparison. * $\mathrm{p}<0.05 ; * * \mathrm{p}<0.01$; *** $\mathrm{p}<0.001$.

\section{In vivo anticancer activity.}

Based on their in vitro profile, we tested $\mathbf{E 5}$ and $\mathbf{E 7}$ in appropriate animal models. We first performed pharmacokinetic (PK) studies on healthy mice to determine whether the compounds would show suitable PK. Initial PK data were obtained using intravenous (i.v.) administration of E5 and E7 (Supplementary Material and Table S16). E7 showed the best PK profile (Fig.8A). Both after i.v. and oral administration it reached its $t_{\max }$ value after about 5 min with plasma levels in the micromolar range, i.e. concentrations that had caused a cell-growth inhibition in vitro. With a half-life of 13.6 hours, a prolonged exposure of the target tissue to the drug is expected. E7 was then selected to be tested for its antitumor effect in vivo. In general, for low molecular weight compounds, intraperitoneal (i.p.) and i.v. administrations result in comparable PK. Furthermore, 
because of the lipophilicity of the compound, a rapid transfer from the peritoneal cavity to the blood and back is expected. Since we preferred to analyze the antitumor activity of E7 in a PDAC orthotopic model that recapitulates several human-tumor phenotypic characteristics, we chose an i.p. drug delivery with an intermittent schedule (Fig.8B). Such a relatively frequent schedule would have been too stressful for the mice via the i.v. route because of the damage to veins. The ability of the hTS dimer disrupter $\mathbf{E} 7$ to inhibit tumor growth was evaluated on bioluminescent PDAC models genetically engineered to express Gaussia-luciferase (G-luc), simplifying the monitoring of the tumor volume (Supplementary Material, Fig.8C). Five days after injection, primary pancreatic tumors were detectable in all mice (100\% take rate, without surgery-related mortality), and G-luc activity, proportional to the number of cancer cells, increased with time (Fig. 8C). This mean signal was significantly lower in mice treated with E7 compared with control mice ( $46183 \pm 2015$ vs. $67905 \pm 6159$ RLU/sec at day $25 ; \mathrm{p}=0.001$, two-sided $\mathrm{t}$ test). Moreover, starting from day 30, the group receiving E7 had significantly lower G-luc intensity compared with mice receiving 5-FU (i.e., at day $30,-70 \%$ and $-48 \%$, relative to control mice, respectively). Mice treated with $\mathbf{E 7}$ had only a slight reduction of body weight (Fig. 8D), similar or less evident than that with 5-FU and showed no other side effects. Tumor growth inhibition was reflected in a significantly longer survival of mice treated with $\mathbf{E} 7$ as compared with control mice ( $p=0.03$, Fig. 8E) and 5FU (58 days, 43.3 days and 50 days respectively). To demonstrate that the therapeutic effect of $\mathbf{E} 7$ is not cancer model specific, $2 \times 10^{6}$ human ovarian cancer cells (A2780) were subcutaneously xenotransplanted into the flanks of 7-week-old Athymic Nude-Foxn1nu female mice (Fig. S21). Seven days after injection, as for the orthotopic model, the engrafted mice were randomly divided into three experimental groups and then treated with a similar drug schedule and dose. We found that also in this model, E7 caused a significant reduction of tumor growth compared with control 
mice $\left(596.1 \pm 167.7\right.$ vs. $2319.9 \pm 289.7 \mathrm{~mm}^{3}$ at day $6 ; 776.5 \pm 191.6$ vs. $3102.0 \pm 516.2 \mathrm{~mm}^{3}$ at day 7 ; $p<0.05$, two-sided $t$ test), which was confirmed by quantification of the area under curve and resulted in significantly longer survival ( $\mathrm{p}=0.0111 ; 24$ days $v s 16$ days; Fig. S21).

To understand whether the antitumor activity of $\mathbf{E 7}$ was associated with an increased anti-TS effect, we measured hTS mRNA and protein expression in tumor tissues from the PDAC orthotopic model (Fig. 8F and Fig. 8G). The mice treated with 5-FU did not show a decrease in TS mRNA expression and showed a more intense staining for TS, in agreement with earlier studies in which treatment with $\mathbf{5 - F U}$ had induced TS activity both in mice and patients $(27,28)$. Remarkably, in complete agreement with the results obtained with model cancer cells, E7 treatment led to a reduced expression of TS mRNA in vivo, down to 40-50\%. Immunohistochemistry staining showed that E7 caused a decrease in the levels of hTS protein that was even more pronounced than 5-FU (Figs. 8F and 8G). The hTS protein levels measured with the histological score (H-score) was $48 \pm 5$ a.u. for $\mathbf{E 7}$ vs. $242 \pm 26$ a.u. for $\mathbf{5}-\mathbf{F U}$ (p<0.001, twosided t test) (29). These results provide clear evidence of the higher anticancer efficacy of E7 relative to 5-FU in the cancer animal models employed; moreover, they support our hypothesis on of the mechanisms of action of these compounds in cells and in tissues. 

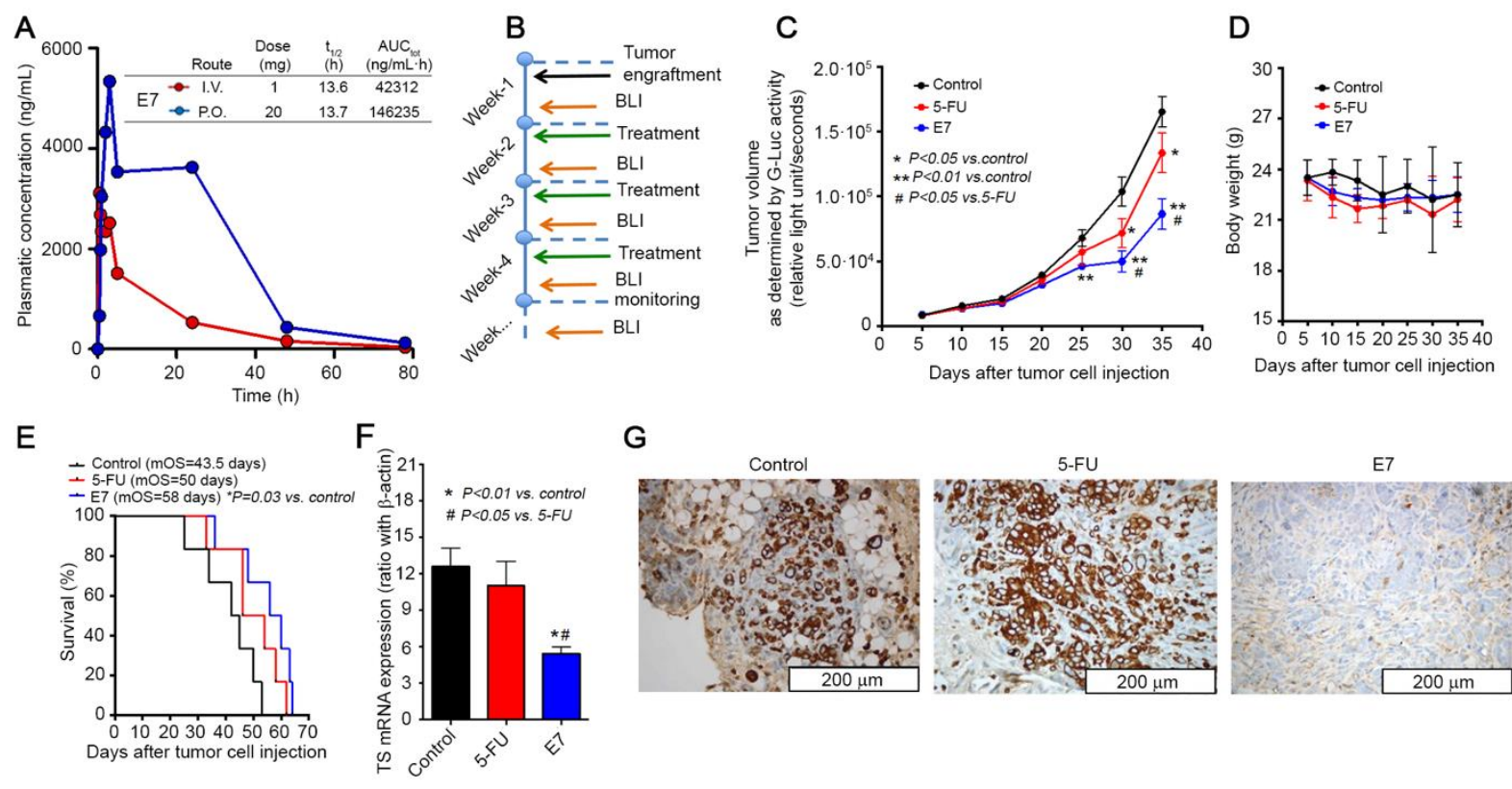

G
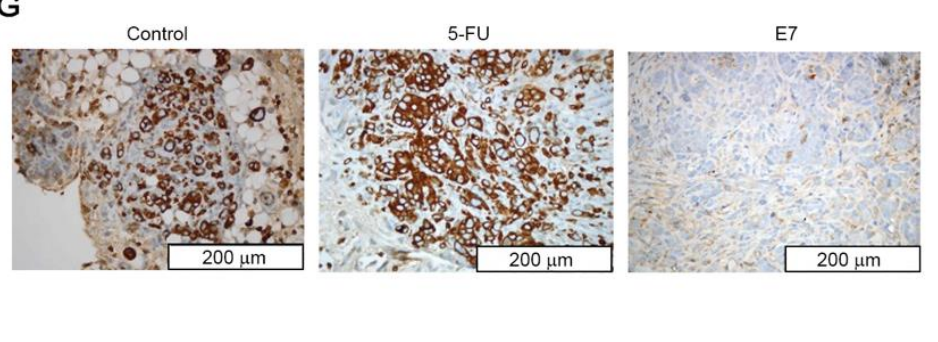

Figure 8. Effects of E7 in vivo. A, PK data on healthy mice for E7 (similar data were observed for E5). B, Set-up of in vivo experiments, using mice injected orthotopically with PDAC-2-primary cells, and monitored every 5 days by bioluminescence imaging (BLI). C, Effects of E7 and 5-FU on tumor growth, as detected in blood samples with the G-luc (proportional to the number of cancer cells, carrying G-luc). Points indicate mean values obtained from the analysis of the six mice in each group. Bars indicate standard deviation. P values were calculated with two-sided Student's $t$ test and ANOVA followed by the Tukey's multiple comparison. D, Effects of E7 and 5-FU on the weight of the mice, demonstrating that tumor shrinkage induced by these treatments was not accompanied by severe toxicity. Points indicate mean values obtained from the analysis of the six mice in each group. E, Survival curves in the groups of mice treated with E7 and 5-FU. Median overall survival (mos) is reported. Statistically significant differences were determined by twosided log-rank test. F, Quantitative PCR results (calculated with the standard curve method, using the ratio with the housekeeping gene $\beta$-actin) showing the reduced expression of hTS mRNA in lysates from frozen tissues from PDAC-2 mice treated with E7 (24 hours before their sacrifice) 
compared with untreated control mice. Columns and bars indicate mean values and standard deviation. $\mathrm{P}$ values were calculated with two-sided Student $\mathrm{t}$ test and ANOVA followed by the Tukey's multiple comparison. Representative immunohistochemical images showing a weak staining for hTS in the tissues from mice treated with E7 compared to the strong staining in 5-FUtreated and control mice.

\section{Discussion}

The small molecules identified in the present work can shift the hTS dimer-monomer equilibrium to the monomer leading to dimer disruption, thus inhibiting the activity of the enzyme and markedly enhancing its proteasomal degradation. The important consequences are the reduction of the intracellular levels of the enzyme and a marked decrease of both in vitro cancer cell growth and in vivo tumor growth. Therefore, these dimer disrupters represent new, promising tools to fight drug resistance associated with high TS levels in different tumor types such as colorectal, ovarian and pancreatic cancers.

Measurement of FRET between two reporter probes bound to the recombinant enzyme showed excitation-energy transfer efficiency to decrease as a result of treatment with compounds able to bind the protein and destabilize its dimeric assembly in favor of the monomers. We measured larger dimer/monomer dissociation equilibrium constants when the protein was saturated by ligands C2-C4 than with the free enzyme. A mechanistic model (Fig.4E) with a single set of equilibrium constants provided a remarkably consistent quantitative description of both spectroscopic (FRET) and kinetic (inhibition) results. However, because compounds C2-C4 did not show good inhibition of cancer cell growth, likely because of their poor cellular uptake, we focused on more lipophilic derivatives with similar enzyme inhibition and FRET profiles but much 
higher biological activity, E5 and E7. These compounds induced apoptotic cell death, thus inhibiting the growth of cancer cells both sensitive and resistant to 5-FU or cisplatin, with similar potency. Such cytotoxicities were mediated by the interaction of these compounds with hTS consistently with the key observation of their intracellular engagement of hTS in a microscopically monitored FRET between an E5-bound excitation energy donor (FITC) and the hTS-tetracysbound rhodamine acceptor (ReAsH).

We have shown that a crucial consequence of the intracellular hTS dimer-to-monomer switch is the enhancement of its proteasomal degradation, an outcome that can be of great advantage in therapeutic applications. The biological consequence of the dimer-to-monomer equilibrium shift were made evident with cells that ectopically expressed the essentially monomeric hTS F59A interface mutant: we observed a markedly accelerated enzyme turnover relative to WT (dimeric) hTS-expressing cells, a finding that can be of large relevance for the development of therapeutic schemes aiming at avoiding hTS over-expression.

Compound E7 exhibited a good pharmacokinetic profile in healthy mice and, when tested on a pancreatic-tumor orthotopic mouse model, showed an in vivo anticancer activity much greater than 5-FU (Fig.7). Additionally, it caused a significantly longer survival with respect to control mice and did not produce toxicity effects. The in vivo markers of $\mathbf{E 7}$ anticancer activity were similar to those observed in in vitro cancer cells, i.e., an immunohistochemically determined large decrease of hTS levels in the analyzed tissues. This is at variance with the increase in the hTS levels induced by treatment with 5-FU. The superior in vivo behavior of $\mathbf{E} 7$ relative to $\mathbf{5 - F U}$, in spite of its similar capacity to inhibit cancer cell growth, confirms its high potential for further development. Our experiments suggest that it may overcome drug resistance in cancer cells that directly or indirectly rise the hTS levels. 
In conclusion, we have proposed a genuinely new class of inhibitors of a critical anti-cancer drug target, hTS. They act as disrupters of the active obligate dimer, resulting in the inactive monomers. With their unprecedented mechanism of inhibition, compounds such as E7 break the longestablished link between inhibition and enhanced expression of this essential enzyme, a link that is typical of classical hTS active-site inhibitors. By doing so, these compounds, once combined with classical anti-TS drugs in suitably designed anticancer therapeutic schemes, may help avoiding or delaying the onset of anti-hTS drug resistance associated with over-expression of the enzyme, thus maintaining or restoring cancer-cell drug sensitivity.

\section{Materials and Methods}

Additional information is provided in SI Materials and Methods.

\section{Reference and note}

1. L. Taddia, D. D'Arca, S. Ferrari, C. Marraccini, L. Severi, G. Ponterini, Y.G. Assaraf, G. Marverti, M. P. Costi, Inside the biochemical pathways of thymidylate synthase perturbed by anticancer drugs: Novel strategies to overcome cancer chemoresistance. Drug Resistance Updates 23, 20-54 (2015).

2. F. Genovese, S. Ferrari, G. Guaitoli, M. Caselli M. P. Costi, G. Ponterini, Dimer-monomer equilibrium of human thymidylate synthase monitored by fluorescence resonance energy transfer. Protein Sci. 19, 1023-1030 (2010).

3. M. P. Costi, S. Ferrari, A. Venturelli, S. Calò, D. Tondi, D. Barlocco, Thymidylate synthase structure, function and implication in drug discovery. Curr. Med. Chem. 12, 2241-2258 (2005).

4. C. W. Carreras, D. V. Santi, The catalytic mechanism and structure of thymidylate synthase. Annu. Rev. Biochem. 64, 721-762 (1995). 
5. N. D. Brunn, S. M. Dibrov, M. B. Kao, M. Ghassemian, T. Hermann, Analysis of mRNA recognition by human thymidylate synthase. Biosci. Rep. 34, e00168 (2014).

6. B. A. Jennings, G. Willis, How folate metabolism affects colorectal cancer development and treatment; a story of heterogeneity and pleiotropy. Cancer Letters 356, 224-230 (2015).

7. Peters GJ. Cancer drug resistance: a new perspective. Cancer Drug Resist 1, 1-5 (2018).

8. D. M. Voeller, M. Zajac-Kaye, R. J. Fisher, C. J. Allegra, The identification of thymidylate synthase peptide domains located in the interface region that bind thymidylate synthase mRNA. Biochem. Biophys. Res. Comm. 297, 24-31 (2002).

9. D. Cardinale, G. Guaitoli, D. Tondi, R. Luciani, S. Henrich, O. M. H. Salo-Ahen, S. Ferrari, G. Marverti, D. Guerrieri, A. Ligabue, C. Frassineti, C. Pozzi, S. Mangani, D. Fessas, R. Guerrini, G. Ponterini, R. C. Wade, M.P Costi, Protein-protein interface-binding peptides inhibit the cancer therapy target human thymidylate synthase. Proc. Natl. Acad. Sci. U SA 108, E542-E549 (2011). 10. D. A. Erlanson, A. C. Braisted, D. R. Raphael, M. Randal, R. M. Stroud, E. M. Gordon, J. A. Wells, Site-directed ligand discovery. Proc. Natl. Acad. Sci. USA 97, 9367-9372 (2000).

11. S. G. Kathman, A. V. Statsyuk, Covalent tethering of fragments for covalent probe discovery. Med. Chem. Comm. 7, 576-585 (2016).

12. O.M. Salo-Ahen, A. Tochowicz, C. Pozzi, D. Cardinale, S. Ferrari, Y. Boum, S. Mangani, R. M Stroud, P. Saxena, H. Myllykallio, M. P. Costi, G. Ponterini, R. C Wade. Hotspots in an obligate homodimeric anticancer target. Structural and functional effects of interfacial mutations in human thymidylate synthase. J. Med. Chem. 58, 3572-3581 (2015).

13. J. Phan, S. Koli, W. Minor, R. B. Dunlap, S. H. Berger, L. Lebioda, Human thymidylate synthase is in the closed conformation when complexed with dUMP and raltitrexed, an antifolate drug. Biochemistry 40, 1897-1902 (2001). 
14. J. J. Irwin, B. K. Shoichet, ZINC - A free database of commercially available compounds for virtual screening. J. Chem. Inf. Model. 45, 177-182 (2005).

15. S. Cross, M. Baroni, E. Carosati, P. Benedetti, S. Clementi, FLAP: GRID molecular interaction fields in virtual screening. Validation using the DUD data set. J. Chem. Inf. Model. 50, 1442-1450 (2010).

16. G. Ponterini, A. Martello, G. Pavesi, A. Lauriola, R. Luciani, M. Santucci, M. Pelà, G. Gozzi, S. Pacifico, R. Guerrini, G. Marverti, M. P. Costi, D. D'Arca, Intracellular quantitative detection of human thymidylate synthase engagement with an unconventional inhibitor using tetracysteinediarsenical-probe technology. Sci. Reports 6, 27198 (2016).

17. A. Avan, V. Caretti, N. Funel, E. Galvani, M. Maftouh, R. J Honeywell, T. Lagerweij, O. Van Tellingen, D. Campani, D. Fuchs, H. M Verheul, G.-Jan Schuurhuis, U. Boggi, G. J Peters, T. Würdinger, E. Giovannetti. Crizotinib inhibits metabolic inactivation of gemcitabine in c-Metdriven pancreatic carcinoma. Cancer Res.73, 6745-56 (2013).

18. K. Bracht, A. M. Nicholls, Y. Liu, W. F Bodmer. 5-Fluorouracil response in a large panel of colorectal cancer cell lines is associated with mismatch repair deficiency. Br. J. Cancer 103, 340346 (2010).

19. E. Chu, D. M. Koeller, J. L. Casey, J. C. Drake, B. A. Chabner, P. C. Elwood, S. Zinn, C. J. Allegra, Autoregulation of human thymidylate synthase messenger RNA translation by thymidylate synthase. Proc. Natl. Acad. Sci. USA 88, 8977-8981 (1991).

20. S. H. Berger, F. G. Berger, L. Lebioda, Effects of ligand binding and conformational switching on intracellular stability of human thymidylate synthase. Biochim. Biophys.Acta 1696, 15- 22 (2004). 
21. M. M. Peña, S. P. Melo, Y.-Y. Xing, K. White, K. W. Barbour, F. G. Berger, The intrinsically disordered $\mathrm{N}$-terminal domain of thymidylate synthase targets the enzyme to the ubiquitinindependent proteasomal degradation pathway. J. Biol. Chem. 284, 31597-31607 (2009).

22. P. M. Wilson, P. V. Danenberg, P. G. Johnston, H. J. Lenz, R. D. Ladner, Standing the test of time: targeting thymidylate biosynthesis in cancer therapy. Nat. Rev. Clin. Oncol. 11, 282-298 (2014).

23. M. E. Kitchens, A. M. Forsthoefel, K. W. Barbour, H. T. Spencer, F. G. Berger, Mechanisms of acquired resistance to thymidylate synthase inhibitors: the role of enzyme stability. Mol. Pharmacol. 56, 1063-1070 (1999).

24. M. E. Kitchens, A. M. Forsthoefel, Z. Rafique, H. T. Spencer, F. G. Berger, Ligand mediated induction of thymidylate occurs by enzyme stabilization. J. Biol. Chem.274, 12544-12547 (1999). 25. J-M. Shi, J. Pei, E-Q. Liu, L. Zhang, Bis(sulfosuccinimidyl) suberate (BS3) crosslinking analysis of the behavior of amyloid- $\beta$ peptide in solution and in phospholipid membranes. PLoS ONE 12(3), e0173871 (2017). https://doi.org/10.1371/journal.pone.0173871

26. G. Mei, A. Di Venere, N. Rosato, A. Finazzi-Agro, The importance of being dimeric, FEBS Journal 272, 16-27 (2005).

27. B. Cellini, R. Montioli, A. Paiardini, A. Lorenzetto, F. Maset, T. Bellini, E. Oppici, C. Borri Voltattorni, Molecular defects of the glycine 41 variants of alanine glyoxylate aminotransferase associated with primary hyperoxaluria type I. PNAS, 107 2896-1 (2010).

28. C. L. Van der Wilt, H. M. Pinedo, K. Smid, G. J. Peters, Elevation of Thymidylate Synthase following 5-Fluorouracil Treatment Is Prevented by the Addition of Leucovorin in Murine Colon Tumors Cancer Res. 52, 4922-8 (1992). 
29. G. J. Peters, G.J. Backus, H.H.J. Freemantle, S. Van Triest, B. Codacci-Pisanelli, G. Van der Wilt, C.L. Smid, K. Lunec, J. Calvert, A.H. Marsh, S. H. L. McLeod, E. Bloemena, S. Meijer, G. Jansen, C. J. van Groeningen, H. M. Pinedo, Induction of thymidylate synthase as a 5-fluorouracil resistance mechanism. Biochim. Biophys.Acta 1587, 194-205 (2002).

Acknowledgments: We thank Dr. Hannu Myllykallio for cloning hTS and preparing bacterial cells expressing mutant proteins, and Dr. Amir Avan and Dr. Niccola Funel for their work on PDAC animal models and stainings. The authors also acknowledge the 'Fondazione Cassa di Risparmio di Modena' for funding the UHPLC-ESI-QTOF system at the Centro Interdipartimentale Grandi Strumenti (CIGS). PP is grateful to Camilla degli Scrovegni for continuous inspirational support.

This work was funded by AIRC2015 IG16977 (MPC), European Union (LIGHTS-A Framework 6 STREP: LSH- 2005-2.2.0-8) Grant agreement n037852 to M.P.C, R.C.W., G.C., R.M.S; CCA foundation grants-2012/2015 CCA2015-1-19 to E.G. and G.J.P., AIRC 14422 Start-Up grant to E.G., the Klaus Tschira Foundation (R.C.W., S.H., O.M.H.S-A) and the Alexander von Humboldt Foundation, the Finnish Cultural Foundation, the Academy of Finland (137918), and the University of Eastern Finland (O.M.H.S-A). RMS is supported by NIH GM24485. PP is supported by Italian Association for Cancer Research (AIRC, IG 23670) and the Italian Ministry of Education, University and Research (PRIN Grant 2017E5L5P3). AR is supported by the Italian Ministry of Health (GR-2016-02364602) and by the Italian Ministry of Education, University and Research (PRIN, Grant 2017XA5J5N). 